\title{
Discourse Networks of Emergency Response Communication: A Case Study of Civilian Deliberation on the Reddit Platform
}

\author{
Cody Blake Wilson \\ Purdue University \\ Indiana, West Lafayette U.S.A. \\ Megan Kendall \\ Purdue University \\ Indiana, West Lafayette U.S.A.
}

\begin{abstract}
Using discourse and network analysis, this research explores a reddit thread concerning an emergency situation in the U.S.V.I. after a 2017 hurricane. In particular, research seeks to gain greater understanding of deliberation and problem-solving behaviour of a crowdsourced, citizen group in response to a disaster. Findings support the use of network analysis and measures of centrality to isolate particularly important actors and contributions in a network. Furthermore, findings also depict seven archetypical roles played by users in the discourse. Further research is needed to understand how the tier structure of the reddit platform influences network structure and behaviour and normalize methods and approaches to conducting network-based research on the platform for consistent and reliable findings across research cases.
\end{abstract}

Keywords — Emergency, Deliberation, Discourse, Networks, Social media

SUGGESTED CITATION: Wilson, C. B., \& Kendall, M. (2018). Discourse networks of emergency response communication: A case study of civilian deliberation on the Reddit platform. Proceedings of the International Crisis and Risk Communication Conference (pp. 12-15). Orlando, FL, USA. Nicholson School of Communication. https://doi.org/10.30658/icrcc.2018.4

\section{INTRODUCTION}

In the last few years, millions of global citizens have experienced at least one catastrophic-level event. Whether it was a flood, tsunami, hurricane, fire, tornado, earthquake, mass shooting, or other terrorist event, timely and effective emergency communication can be lifesaving. Government agencies tasked with emergency management functions typically deliver this kind of communication. However, individuals also turn to other actors in their social networks for information, social, and collaborative support [1]. In the digital age, these networks are often found online through social media platforms such as Twitter, Facebook, and reddit. Thus, when faced with problems in emergency circumstances, these platforms become incredibly useful for those impacted.

This paper explores how one user sought help from a reddit subchannel (pseudonym: /r/HurricaneHelp) in the aftermath of Hurricane Irma. The thread garnered over 46,000 up-votes and over 1,500 comments at the time of data collection. Two predominate questions directed the research: 1) can network analysis be used by emergency managers to target particularly useful actors in a network, and 2) what connections can be made between discourse and network roles in the network? First, literature on the use of social media in emergency circumstances will be reviewed, along with literature concerning discourse behaviours and network theory. Then, discourse and network analysis procedures will be described. Results point to the usefulness of centrality measures to find particularly important actors in the network, rather than traditional reddit measures, such as up-votes or amount of thread contribution. Furthermore, the discoursenetwork analysis combination reveals seven particularly interesting discourse archetypes. Findings provide greater understanding to the academic community of how reddit is used in public deliberation during emergency, as well as the merits of combining discourse and network analysis in social media analytics more broadly. 


\section{LITERATURE REVIEW}

Interdisciplinary scholarship on issues surrounding the management of emergencies has recently ballooned, and top scholars in communication have called for an even stronger focus in this area (Palenchar \& Heath, 2007). Social media and the internet at large has been found to be important for emergency information distribution and deliberation, especially towards the tasks of rescue needs, missing persons or animals, building resilience, and finding resources [2][3].

To understand more about these practices, two pre-existing frameworks for classifying deliberation behaviour were used. The first is a six-part framework built specifically for deliberation in online contexts, proposed by Stromer-Galley [4]. This framework includes: 1) reasoned opinion expression, 2) references to external sources, 3) expressions of disagreements, 4) equal levels of participation, 5) coherence to structure and topic of deliberation, and 6) engagement among participants. This classification scheme is particularly useful for identifying meaningful behaviours in reddit threads, as the structure of the thread allows for easy understanding of the discourse structure. A second classification scheme adds to Stromer-Galley's [4] by highlighting specific problem-solving leadership behaviours in discourse. Tyshchuck, Li, Ji, and Wallace [5] identified three main roles in their study of online emergency problem-solving discourse: 1) dispensing unique, new, and relevant information, 2) providing confirmation or disconfirmation to members of the community, and 3) urging others to take action. Together, both these frameworks provide a means of classifying the communicative role of members in discourse.

Network approaches provide a unique and natural approach to the study of a variety of social phenomena. First, many natural processes (such as discourse) can be viewed as occurring in a network structure. Second, discovering an individual's place within a network sheds insight upon the limitations and opportunities of their behavior. Third, social groups are, at least in part, impacted by the structure of the networks that exist within them [6]. Based on these assumptions, it is reasonable to predict that the deliberation role actors take upon themselves will impact the discourse network surrounding them, leading to correlations between these behaviors and node-level characteristics. However, little research has been done to combine discourse and network analysis in this particular context.

\section{DISCOURSE AND NETWORK ANALYSIS}

The first 24 hours of response to a post seeking help in the subreddit/r/HurricaneHelp after a 2017 hurricane was collected. To do this, the posts were organized chronologically on the webpage, which was then scraped and saved separately. Bots were not included in the data set; neither were posts made by deleted accounts, deleted posts, or the resulting conversation branches out of respect for user privacy. In total, 690 unique comments made by 411 unique users were collected.

Data was stored in a table which captured the sender, the receiver, the up-vote count, the text, and the "tier" of each comment. Comments responding directly to the original post were considered "tier 1." Comments on tier 1 comments were coded as "tier 2." Thus, the "deeper" comments were in the discourse, the higher their tier.

The comments were then qualitatively coded for their discourse behaviours using Stromer-Gally's [4] and Tyshchuck et al.'s [5] classification schemes. Coders used adapted criteria described in the original articles [4][5]. Because the researcher most concerned with problem-solving behavior surrounding the situation presented in the original post of the network, only comments pertaining to this problem were coded under the leadership framework. Whether or not a comment pertained to the original situation was determined by positive hits of the CST variable of the deliberation content framework. In other words, only comments identified as remaining coherent to the structure and topic of the original conversation were coded for leadership characteristics. Comments were coded dichotomously ( 1 or 0$)$ for all classifications, except for equal level of participation, which was calculated at the comment level by dividing the word count of the comment with the total word count in the network. Then, individual users were coded for their discourse behaviours. For most of the behaviours, users were coded dichotomously (1 or 0). A user was coded for a behaviour as long as they demonstrated it in a least one comment. Equal level of participation was measured at the user level by dividing the total word count of each user by the total word count of the network.

Next, the discourse data was used to construct a network using the NodeXL program [7]. In this particular network, nodes represented users and edges represented comments. Standard network and node-level data was collected, including in-degree (how many unique users commented to a node), out-degree (how many unique users on which a node commented), closeness centrality (how closely connected a node was to a variety of conversations topics), eigenvector centrality (how closely a node was connected to conversation facilitators), reciprocated vertex pair ratio (how likely a node was to engage in dialogue), and clustering coefficient (the degree to which a node deliberated in depth with consistent other nodes).

\section{RESULTS}

Correlation tests between node-level network metrics and the deliberation behaviours comments revealed measures of 
centrality to be most effective for identifying users exhibiting these behaviours. Although up-votes did not correlate with any deliberation behaviours and word count correlated with only reasoned opinion expression, references to external sources, and expressing disagreement $(p<0.01)$, closeness centrality correlated to references to reasoned opinion expression $(p>0.05)$, references to external sources $(p<0.001)$, coherence to structure and topic $(p<0.001)$, information sharing $(p<0.001)$, information (dis)confirmation $(p<0.05)$, and urging action $(p<0.001)$. Eigenvector centrality correlated strongly with reasoned opinion expression $(\mathrm{p}<0.01)$, references to external sources $(\mathrm{p}<0.001)$, coherence to structure and topic $(\mathrm{p}<0.001)$, information sharing $(\mathrm{p}<0.05)$, and urging action $(\mathrm{p}<0.001)$. Other node-level metrics, such as in-degree, out-degree, clustering coefficient, and reciprocated vertex pair-ratio, were not as highly correlated with important deliberation behaviours.

Tier-level was a highly determinative factor of contribution. In total, there existed 19 tiers, with $27.8 \%$ of the comments happening within the first three tiers, 56.5\% happening within the first five tiers, and $77.2 \%$ of comments occurring within the first seven tiers. By tier 8 , less than $50 \%$ of the conversation was coded for reasoned opinion sharing, references to external sources, and expressing disagreement. Furthermore, $0 \%$ of comments beyond tier 7 were found to be coherent to structure and topic of the original problem presented to the thread. A pattern began to emerge within the first tiers concerning problem-solving leadership behaviour. While the frequency of information sharing behaviour fell consistently beyond the first tier, information (dis)confirmation and action urging peaked around the third tier before falling off as well.

Using the results of both the discourse and network analysis, seven archetypical deliberation roles were found amongst participants. These include the Axis, the Facilitator, the Commentator, the Informant, the Commander, the Forensic, and the Fire-Starter. The Axis is a role characterized by high degrees of centrality, involvement, and information sharing. They typically share information about and/or present the problem. Most importantly, the remained involved in the discourse. Facilitators are also highly central and act as bridges between different network clusters. These clusters often represent different directions of conversation; thus, the facilitator often takes on the task of directing many different conversations towards solutions. Commentators are also highly central but tend to share reasoned opinions and disagreement more frequently than other behaviours. Commentators act as the "devil's advocates" of the discourse. Informants are highly central but contribute very little. They are not typically involved in the discourse outside of sharing important and valuable information. There contributions, however, tend to create lots of conversation as a result of their contributions. Commanders are most focused on action. Like Facilitators, they bridge clusters. However, they also almost exclusively report on their own actions and urge others to take action as well. Forensics are often segmented to one particular cluster, and their behaviours tend to be mostly information sharing and (dis)confirmation. Lastly, Fire-Starters, like Informants, tend not to post a lot in the discourse. However, their comments are often seen as controversial or offensive, so much so that it greatly influences the nature of the discourse network. Unlike a "troll" though, Fire-Starters comments are still classified as coherent to the problem at hand.

\section{DISCUSSION}

This case study sought to explore how Reddit was utilized for communication by public individuals in response to an emergency situation. Utilizing network and discourse analysis, this study answered two questions: 1) what deliberation and problems solving roles emerge in the discourse network, and 2) how can network and node-level metrics help emergency managers identify certain actors or roles in the network? Using network metrics and coding schemes for identifying deliberation and problem-solving leadership behavior, analysis revealed separate actors that fulfilled roles concerned with eliciting, providing, filtering, and acting on information, as well as roles concerned with focusing, managing, and initiating discourse. These findings mirror and add to previous research concerning online discourses in response to emergency situations. Network and node metrics also revealed closeness and eigenvector as more useful than traditional Reddit-based metrics (number of comments, word count, and up-vote) in determining and isolating important actors and information. These findings can be useful research into the roles individuals play during online deliberation, especially in emergency situations. Findings also support the notion that measures of centrality are some of the best tools for identifying key actors in networks, especially when compared to how individual platforms organize or prioritize actors and contributions. Methodologically, this project further promotes the combination of network science and the communication discipline. By combining two methods, greater understanding was gained over the case than from what could have been gained from either on its own.

\section{CONCLUSION}

This research intended to gain greater understanding of online emergency deliberation behaviors of the general public, as well as the usefulness of network science in this kind of inquiry. Future research concerning this project may include formalizing methodologies and greater study of the tier influence on the network. A formal standard for network analysis of reddit discourse does not yet exist. Such a standard is important, considering the tiered nature of the reddit userinterface and the ability for researchers and practitioners alike to confidently draw transferable meaning from research. 
Furthermore, more research is needed to understand exactly how the tier nature of the user-interface impacts both human behavior and network structure. Future research in this area may include sequence analysis, user-computer interaction analysis, and graph theory. Overall, continued study is needed to better understand and use public deliberation and problem-solving online in emergency situations.

\section{Author Biography}

Cody Blake Wilson earned his M.S. in Communication from the Brian Lamb School of Communication in 2018. He now works in operations and administration for the KIPP Public Charter School network in Indianapolis, IN. He may be reached at wilso248@purdue.edu.

Megan Kendall earned her M.A. in Communication from the Brian Lamb School of Communication in 2018. She is now moving back into professional public relations practice. She may be reached at mkendall@purdue.edu.

\section{REFERENCES}

[1] Vieweg, S., Hughes, A. L., Starbird, K., \& Palen, L. (2010). Microblogging during two natural hazards events: What Twitter may contribute to situational awareness. Computer-Human Interaction 2010: Crisis Informatics (pp. 1079-1088). Atlanta: Association for Computing Machinery.

[2] Macias, W., Hilyard, K., \& Freimuth, V. (2009). Blog functions as risk and crisis communication during Hurricane Katrina. Journal of Computer-Mediated Communication, 15, 1-31. https://doi.org/10.1111/j.1083-6101.2009.01490.x

[3] Thelwall, M., \& Stuart, D. (2007). RUOK? Blogging communication technologies during crises. Journal of ComputerMediated Communication, 12, 523-548. https://doi.org/10.1111/j.1083-6101.2007.00336.x

[4] Stromer-Galley, J. (2007). Measuring deliberation's content: A coding scheme. Journal of Public Deliberation, 3(1), art. 12. Available at: https://www.publicdeliberation.net/jpd/vol3/iss1/art12

[5] Tyshchuk, Y., Li, H., Ji, H., \& Wallace, W. A. (2014). The emergence of communities and their leaders on Twitter following an extreme event. In R. Missaoui, \& I. Sarr (Eds.), Social Network Analysis - Community Detection and Evolution (pp. 1-25). Switzerland: Springer International Publishing. https://doi.org/10.1007/9783-319-12188-8_1

[6] Borgatti, S. P., Everett, M. G., \& Johnson, J. C. (2013). Analyzing social networks. London: Sage.

[7] Smith, M., A., C., Milic-Frayling, N., Shneiderman, B., Mendes Rodrigues, E., Leskovec, J., \& Dunne, C. (2010). NodeXL: a free and open network overview, discovery and exploration add-in for Excel 2007/2010/2013/2016. Social Media Research Foundation. 